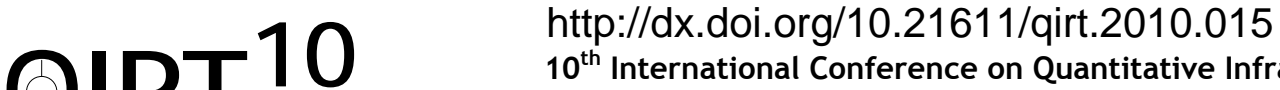 \\ $10^{\text {th }}$ International Conference on Quantitative InfraRed Thermography \\ July 27-30, 2010, Québec (Canada)
}

\section{Active thermography data processing for the NDT\&E of frescoes}

\author{
by A. Bendada*, S. Sfarra**, D. Ambrosini**, D. Paoletti**, C. Ibarra-Castanedo* and X. P. V. Maldague* \\ *Computer Vision and Systems Laboratory, Department of Electrical and Computer Engineering, 1065, av. de la \\ Médecine, Laval University, Quebec City, Canada, G1V 0A6; \{Bendada, IbarraC, MaldagX\}@gel.ulaval.ca \\ ${ }^{*}$ LAS.E.R. Laboratory, Dep. of Mechanical, Management and Energy Eng. (DIMEG), University of L'Aquila, Loc. \\ Monteluco di Roio, 67100 Roio Poggio (AQ), Italy, \{stefano.sfarra, dario.ambrosini,domenica.paoletti\}@univaq.it
}

\begin{abstract}
In this study, a reproduction of Giotto's fresco "Meeting at Golden Gate" containing artificial defects typical of fresco decay was inspected by active infrared thermography. Data was processed using different techniques in order to improve the infrared signature of the defects and to reduce the impact of non-uniform heating and emissivity variations due to the painting's pigments. It is shown that active thermography constitutes an interesting alternative for the in situ inspection of frescoes without impairing their integrity. More research should be carried out to optimize the way that heat is delivered to the surface to improve defect detectability.
\end{abstract}

\section{Introduction}

Frescoes are more than ever subjected to variations in environmental parameters and to other external causes that could damage their integrity. Air conditioning, lightning, air pollutants and increased human contact have strongly accelerated the deterioration of cultural heritage material [1], [2]. For instance, water vapour generated by visitors of a building could condense on walls having a sufficiently low surface temperature [2]. Constant monitoring (moisture on the building and microclimate condition) is thus required in order to diagnose and preserve the decay status of artworks over the years. Many of the diagnostic techniques commonly used nowadays involve the removal of components, which could impair the integrity of the inspected piece. The application of nondestructive testing and evaluation (NDT\&E) techniques on frescoes is therefore highly desirable and could be a turning point in the restoration field.

Thermal non-homogeneities can be caused by a number of frescoes' surface characteristics such as chromatic alterations (rose-coloured areas caused by the presence of bacteria), black crusts (blackening of the surface), salts, material type, colors and decorations, which complicate the proper detection of defects. Nevertheless, advanced signal processing techniques allow to detect and quantify defects (delaminations) using solar radiation in a short ( $\sim 3 \mathrm{~h}$ or less) period of time.

A substantial amount of efforts have been dedicated to the development of suitable active thermography inspection techniques for the assessment of frescos. Grinzato et al. (1994) [3] carried out an interesting study to detect delaminations on laboratory fresco samples using pulsed thermography and proposed a 2D numerical model to study a quantitative approach. A few years later (2000), the same authors proposed [4] a 3D numerical model combined with a 3D adaptive filter to consider the effect of lateral heating considerably increasing the signal-to-noise ratio and consequently improving defect detection and characterization. On a parallel investigation by Grinzato et al. (2000), it has been demonstrated [1] that an approach combining computational fluid dynamics (CFD) and passive thermography could be used to assess the integrity of artworks on historical buildings by measuring the air's relative humidity, velocity and temperature. CFD is used in this case to continuously map the surface temperature for proper indoor air control with reasonable inspection times (a $1 \mathrm{~h}$ data acquisition is sufficient according to [2]). Carlomagno and Meola (2001, 2002) [5],[6] present several examples of detection of detachments, cracks, tiles of different materials, tile disbonds and false tiles on frescoes using lock-in thermography. Ludwig and Rosina (2005) [7] were able to identify improperly restored areas in the Danza Macabra, a medieval external fresco at the Disciplini Oratory building in Clusone, Italy. More recently (2008), quantitative pulsed phase thermography was used to characterize subsurface defects on a laboratory fresco specimen [8].

In this study, a reproduction of Giotto's "Meeting at Golden Gate" was inspected by square heating infrared thermography. This model was constructed [9] based on the use of the same executive techniques and materials of the past, and contains artificial defects typical of fresco decay: static (detachments, cracks, flaking) and dynamic defects (humidity, efflorescences, which is the whitening of the painting layer resulting from salt crystallization on the surface [24]). One of the main difficulties for the application of active thermography techniques for the inspection of frescoes is the strong influence of the different emissivity of the pigments that could led to the misinterpretation of the resulting thermograms. The collected data was processed using several processing techniques with the purpose of detecting and improving the defects' infrared signatures. 


\section{Experimental setup and model description}

The investigated specimen corresponds to a reproduction of Giotto's "Meeting at Golden Gate" (see Fig. 1a) located at the Scrovegni Chapel, in Padua, Italy. The $60 \times 60 \mathrm{~cm}^{2}$ plastered model supported by a wooden frame, shown in Fig. $1 \mathrm{~b}$, was designed and constructed using traditional techniques and materials [9]. Several defects representing wall painting damages typical of fresco decay were included at different layers as illustrated in Fig. 1c.

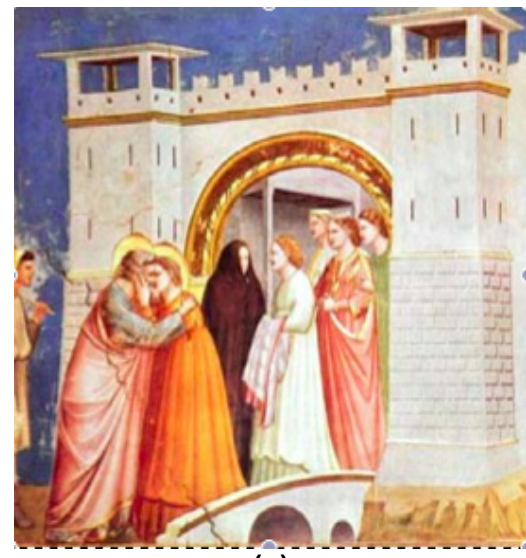

(a)

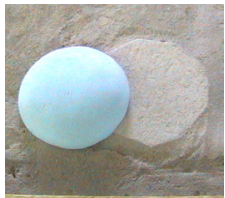

(d)

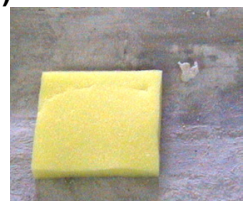

(e)

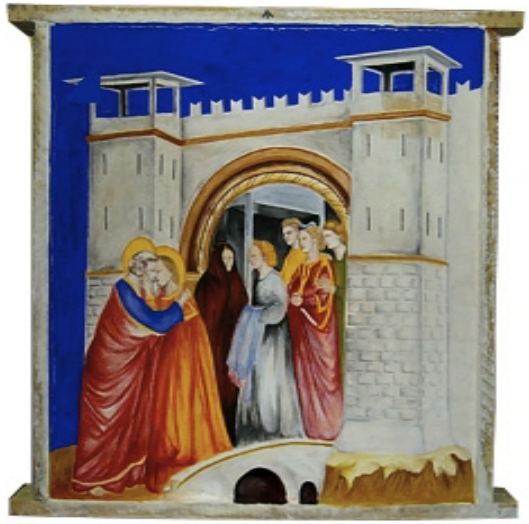

(b)

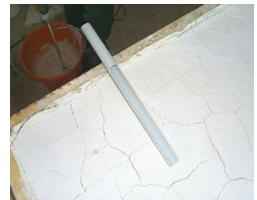

(f)

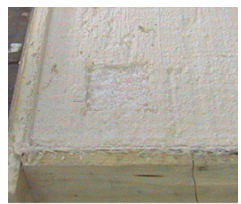

(g)

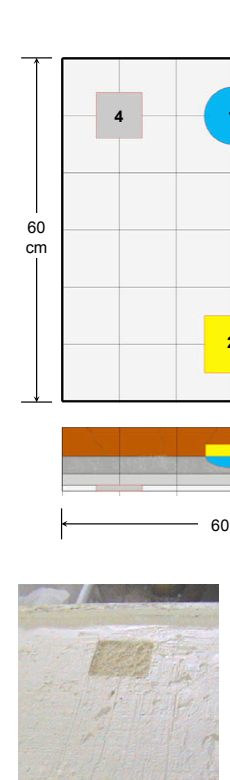

(h)

Fig. 1. (a) Photograph of the original Giotto's fresco "Meeting at Golden Gate"; (b) experimental model with fabricated defects; (c) map of defects; (d) defect 1: void (fabricated with a polystyrene cap); (e) defect 2: sponge; (f) defect 3: painting coat detachment (produced by a half rubber nose); (g) defect 4: efflorescence; (h) defect 5: cracks; and (i) defect 6: flaking.

Details about the different fabricated defects are shown from Fig. 1d to Fig. 1i. Defect 1 (Fig. 1d) is a void, induced by a polystyrene spherical cap $10 \mathrm{~cm}$ wide and $2 \mathrm{~cm}$ thick in shape, which was removed after drying to simulate a detachment of the plaster from the underlying wall. Defect 2 (Fig. 1e) is a $9.3 \times 10 \mathrm{~cm}^{2}$ sponge (at the same depth as defect 1) for which a mobile brick was placed at the back, so as to be extractable to soak the sponge during the experiments. Defect 3 (Fig. 1f) is a half rubber hose $(1 \mathrm{~cm}$ of thickness) inserted through a hole made in the wooden frame creating a void to simulate a detachment between the painting coat and the final skim coat. Defect 4 (Fig. 1g) was designed placing sodium chloride into an $8 \mathrm{~cm}^{2}$ cavity of the final skim coat in order to simulate the weathering by salts (efflorescences). The porous mortar backing provides an easy route for the movement of dilute salt solutions. The volumetric expansion associated with efflorescences crystallization disrupts the plaster-pigment adhesion and leads to the powdering. Defect 5 (Fig. 1h) was made using fine river sand $(2-3 \mathrm{~mm})$ in order to produce cracks of the surface. Defect 6 (Fig. 1i) was produced by mixing polyacetatevinyl with pigments ( 1 volume of polymer, 1 volume of pigment and water) to simulate the detachment of the paint layer (flaking). After drying, the flaking was observed in small areas.

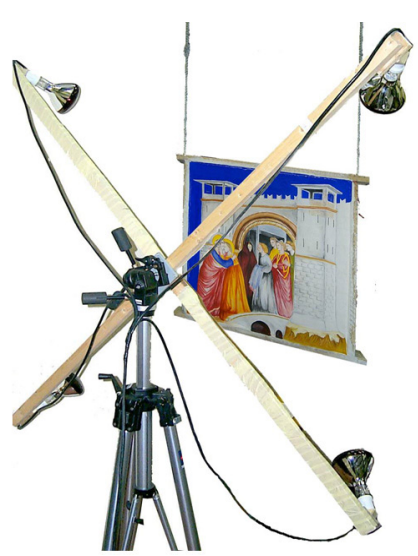

Fig. 2. Experimental setup.

The thermal stimulation is obtained in reflection mode by means of four infrared lamps (OSRAM SICCATHERM, $250 \mathrm{~W}$ ), placed on a wooden frame that also held the IR camera (FLIR S65 HS, 7.5-13 $\mu \mathrm{m}, 320 \times 256)$ as depicted in Fig. 2. The specimen was heated during 2 minutes and then cooled down for about 5 minutes. The whole process was recorded at a frame rate of $1 \mathrm{~Hz}$ for later processing. Defect 2, the sponge (Fig. 1e), has been soaked with $50 \mathrm{~g}$ of water just before the heating and the thermographic recording is initiated. 


\section{Data processing}

Active thermography is a well-know nondestructive testing and evaluation (NDT\&E) technique. The basic principle, experimental configuration and advancements are well documented in the literature [10]-[21]. For sake of simplicity, only a brief review of the technique is provided herein. Interested readers should consult the provided references.

In this study, the specimen surface is stimulated with a heat square pulse and the cooling down process is recorded with an infrared camera for several minutes. The acquired data is then processed to improve defect contrast and signal-tonoise ratio. Several processing techniques exist, from a basic cold image subtraction to more advance techniques such as principle components or higher order statistics. The more relevant to the present study are: differential absolute contrast (DAC) [14], which calculates the thermal contrast of the actual thermal profiles with respect to a reference area that follows the idealized cooling profile dictated by the Fourier's law 1D solution; pulsed phase thermography (PPT) [15],[16] which transforms data from the time domain to the frequency domain in order to obtain phase delay images or phasegrams that have an improved defect contrast; principal component thermography (PCT) [17], which reorganizes into new components that take into account the main spatiotemporal variances of the sequence; thermographic signal reconstruction (TSR) [18],[19], which produces synthetic images (first and second temporal derivatives, phasegrams) from a polynomial regression of raw thermographic data; and higher order statistics (HOS) [20]-[23], which calculates the higher order centralized moments ( $3^{\text {rd }}$ or skewness, $4^{\text {th }}$ or kurtosis, or $n^{\text {th }}$ order moment) of the temporal temperature profiles producing single images summarizing all the relevant information about the original sequence. frescoes.

Next section presents some comparative examples showing the potential of these techniques for the inspection of

\section{Results and discussion}

The mosaic specimen was inspected as described in section 2. A thermogram sequence containing a total of 417 thermograms was available for processing. Fig. 3a presents the raw thermogram showing the maximum thermal contrast for defect 4 (efflorescences, see Fig. 1c and g). Fig. 3b shows the thermal profiles for defect 4 and a sound area next to it. The profiles correspond to the average temperature of a $5 \times 5$ pixels area, i.e. the regions covered by the crosses in Fig. $3 a$. As can be seen, the defect's thermal contrast begins to appear during the heating stage ( 20s) and it slowly drops until it practically disappears at the end of the cooling stage.
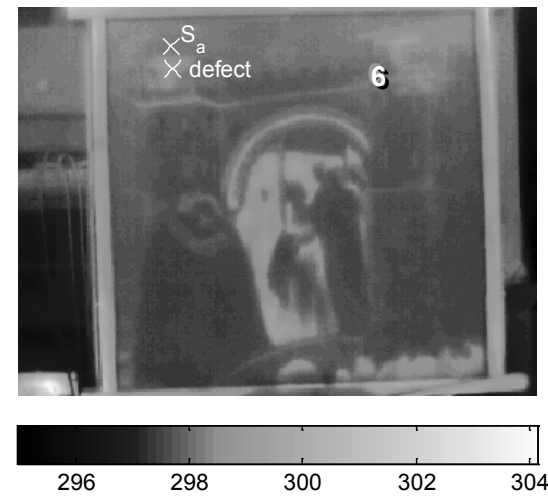

(a)
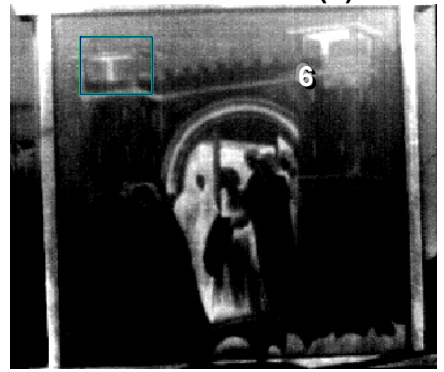

(c)

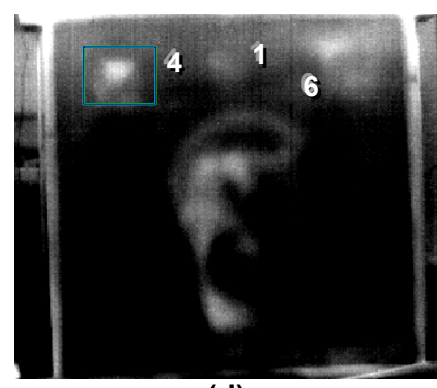

(d)

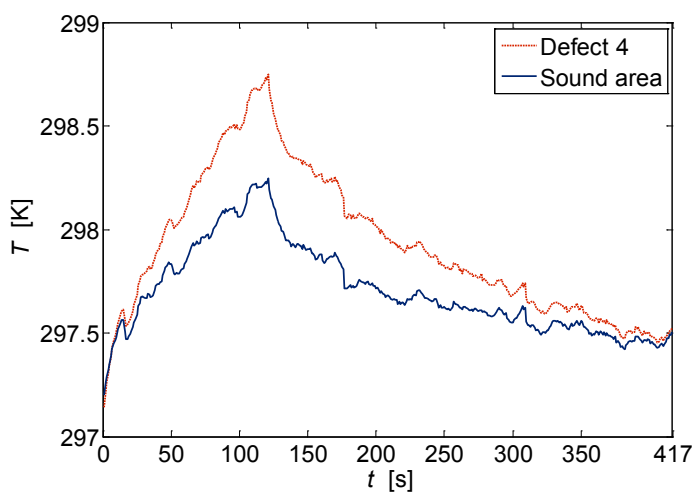

(b)

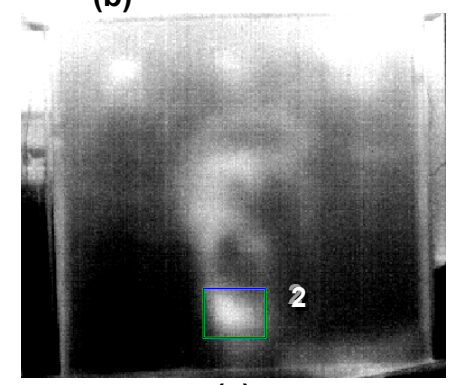

(e)

Fig. 3. (a) Raw thermogram showing maximum contrast for defect 4 at $t=120 \mathrm{~s}$; (b) mean thermal profiles for $5 x 5$ pixels regions (as indicated in the thermogram) for defect 4 and a sound area next to it; raw thermograms at $t=(c) 20 \mathrm{~s}$; (d) $280 \mathrm{~s}$; and (e) $340 \mathrm{~s}$. 
There is also a slight indication of defect 6 (flaking) at early times (see Fig. 3c, right corner), and a weak signal is still present later in the sequence (see Fig. 3d). Defect 1 can also be inferred at later times but with a very weak signal.

The effect of the differences in painting emissivity can be observed in the thermogram of Fig. 3c (see the region of interest delimitated by the rectangle). During the heating phase, the corners of the towers (see also the defect 6 area at the right) emit a strong signal that might preclude the detection of subsurface defects. Nevertheless, at some point during the cooling phase, the thermal signature of the defect begins to be stronger whilst the emission from the painting weakens. In Fig. 3d, defect 4 appears with better contrast. It should be noted however that the top-left corner of defect 4 , which is closer to the left tower corner, presents a higher contrast. This might be caused by a greater concentration of heat in the area because of the emissivity variations or by an uneven distribution of the sodium chloride during the fabrication of the defect. Emissivity variations have an even greater impact on the detection of defect 2. In Fig. $3 \mathrm{e}$ the thermal signature of this defect is observed for a thermogram at a late stage of the cooling phase. The painting area above defect 2 corresponds to white and black robe of the lady standing over the bridge. As was the case for the towers' corners, the black paint produces a very strong thermal signature, especially during the heating phase. As a matter of fact, all the areas painted with dark colors (black or brown) show a stronger emission than the others during heating as seen in Fig. 3c. The impact of dark painting emissions is greatly reduced during cooling, which allows to detect the thermal signature of defect 2.

Advanced processing techniques were used as well in order to improve defect detectability. First, DAC, TSR and PPT were applied to the cooling phase only, i.e. from $t=121 \mathrm{~s}$ to the end, for a total of 297 thermograms, even though, as seen in Fig. 3, some defects produce a detectable contrast during the heating phase. The reason for this is that these three algorithms are based on the assumption that the thermal profiles follow an approximate linear decay on a double-logarithmic scale as predicted by the heat diffusion equation for a 1D [24]. It should be bear in mind however that there is some lost of information from such results. Ideally, the heating phase should be stopped before any defect is visible. For instance, defect 6 would have a better contrast since is the most shallow defect and thus appears very early during the heating stage. Still, results presented in Fig. 4 allowed to detect most of the fabricated defects.

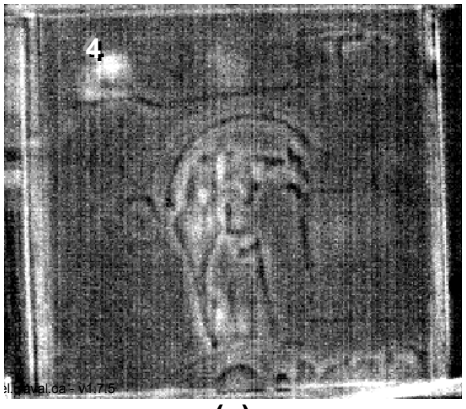

(a)

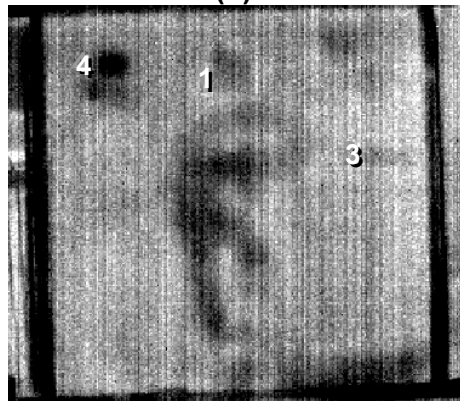

(d)

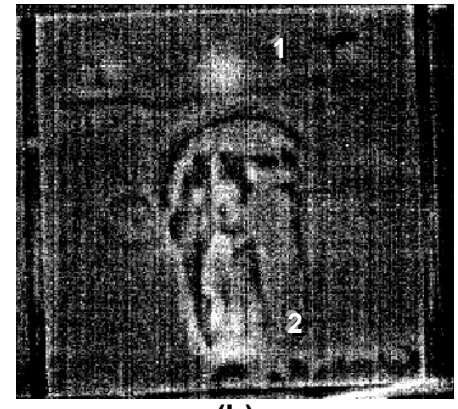

(b)

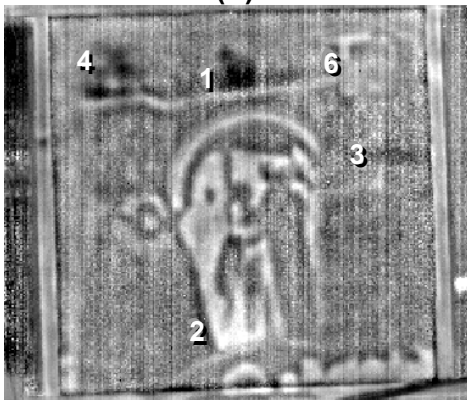

(e)

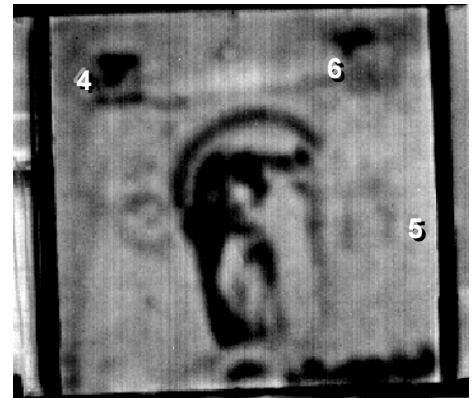

(c)

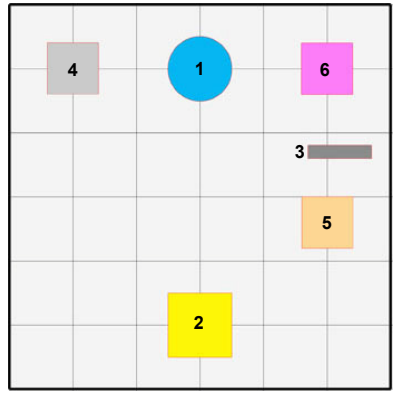

(f)

Fig. 4. (a) $D A C t=48 \mathrm{~s}$, (b) $D A C t=250 \mathrm{~s}$; (c) first time derivative $t=89 \mathrm{~s}$; (d) first time derivative $t=296 \mathrm{~s}$; (e) phasegram $f=3.5 \mathrm{mHz}$; $(f)$ defects map (for reference).

Defects in these images are visible with more or less contrast depending on the processing technique being used. Fig. $4 a$ and $b$ show results obtained from DAC. In the process, a cold image (an image before heating) was subtracted from all thermograms. Defect 4 is visible in Fig. $4 a$ and defects 1 and 2 in Fig. 4b. The first time derivative at two different times allowed the detection of defects 1, 3, 4, 5 and 6 (Fig. 4c and d), whilst defects 1, 2, 3, 4 and 6 are detected in the PPT phasegram of Fig. 4e. The defects map is included in Fig. $4 \mathrm{f}$ for reference.

HOS and PCT were applied to the whole sequence (heating and cooling phases), i.e. 417 thermograms, and some results are presented in Fig. 5. The kurtogram (4th order moment image) in Fig. 5a shows four of the six fabricated defects. 
Interestingly, the contrast is positive or negative with respect to the sound areas in this image which might be used to discriminate between defect types. Furthermore, contrary to all the other techniques, HOS produces a single image result [25].

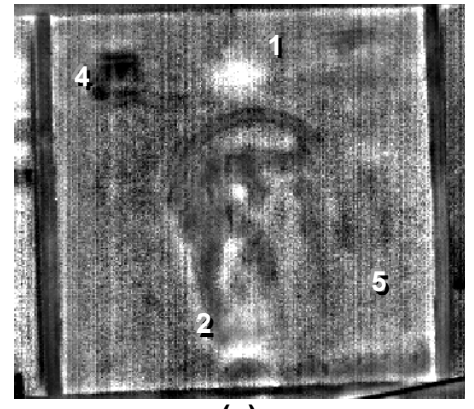

(a)

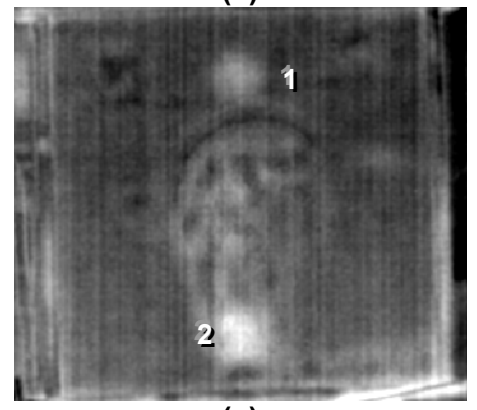

(c)

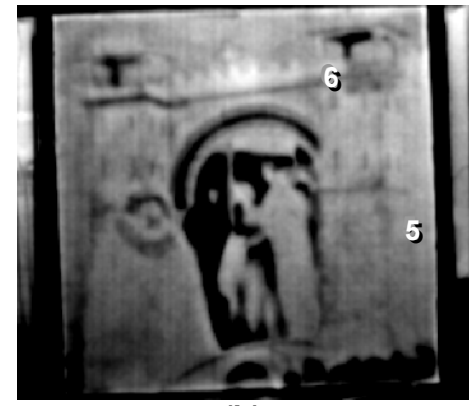

(b)

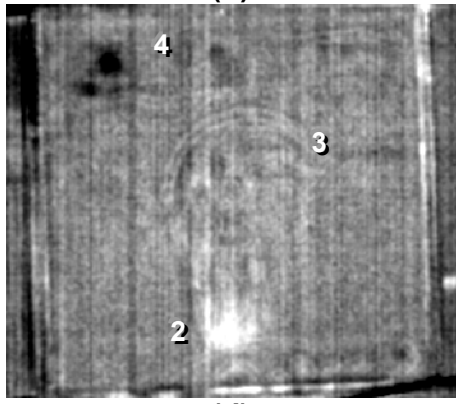

(d)

Fig. 5. Processing results for the whole sequence

(a) kurtogram; and PCT (b) EOF2; (c)

(c) EOF5; $(d)$

(d) EOF6.

PCT results are presented in Fig. $5 b$ to $d$. All defects can be detected with more or less contrast. Defect 2 in particular can be detected with good contrast in Fig. 5c, better than all other processing techniques in Fig. 4 . This is in part due to the fact that the whole sequence was used in the process so more information about the defect was available, contrary to results in Fig. 4 for which only the cooling phase was used.

In all cases, defect 5 appears with a very weak contrast, even though it is located near the surface. Fine river sand was used to fabricate this defect in order to produce cracks in the surface.

\section{Conclusions}

From the results presented herein it can be concluded that the application of active thermography for the in situ NDT\&E of frescoes is very promising. Raw thermographic data allow detecting or at least inferring the existence of 4 of the 6 defects. Nevertheless, advanced signal processing help to improve defect contrast and reduce the impact of both, emissivity variations due to the paintings' infrared signatures and non-uniform heating. Although all defects were detected after processing, defect contrast was still weak for some defects, defect 5 and 3 in particular. There is still room for investigating a more effective way of heating the sample. For instance, using a shorter heat pulse to obtain complete (where defects thermal contrast begins during the cooling stage) thermal profiles for all defects. Another possibility is to use a modulating source (as in lock-in thermography), which would probably be more appropriate in order to assure the integrity of the fresco.

\section{Acknowledgements}

Authors wish to thank Mr. N. Zaccagnini and Eng. R. Di Biase for the fabrication of the fresco and to the Canada Research Program (CRC): Multipolar Infrared Vision Canada Research Chair (MIVIM) for supporting this research.

\section{REFERENCES}

[1] Grinzato E., Bressan C., Peron F., Romagnoni P. and Stevan A. G. "Indoor climatic conditions of ancient buildings by numerical simulation and thermographic measurements," Proceedings of SPIE - The International Society for Optical Engineering, 4020:314-323, 2000. 
[2] Grinzato E., Bressan C., Marinetti S., Bison P.G. and Bonacina C. "Monitoring of the Scrovegni Chapel by IR thermography: Giotto at infrared," Infrared Physics and Technology, 43(3-5):165-169, 2002.

[3] Grinzato E., Bison P., Marinetti S., and Vavilov V. "Nondestructive evaluation of delaminations in Fresco plaster using transient infrared thermography," Research in Nondestructive Evaluation, 5(4):257-274, 1994.

[4] Grinzato E. G., Bison P. G., Marinetti S., Vavilov V., "Thermal NDE enhanced by 3D numerical modeling applied to works of art," $15^{\text {th }}$ WCNDT - World Conf. on Non Destructive Testing, [available online: http://www.ndt.net/article/wcndt00/papers/idn909/idn909.htm], Roma (Italy) 15-21 Oct. 2000.

[5] Carlomagno G. M. and Meola C. "Infrared thermography in the restoration of cultural properties," Proceedings of SPIE - The International Society for Optical Engineering, 4360:203-216, 2001.

[6] Carlomagno G. M. and Meola C. "Comparison between thermographic techniques for frescoes NDT," NDT and E International, 35(8):559-565, 2002.

[7] Ludwig N. and Rosina E. "Dynamic IRT for the frescoes assessment, the study case of Danza Macabra in Clusone (Italy)," Proceedings of SPIE - The International Society for Optical Engineering Thermosense XXVII, 5782:272-279, 2005.

[8] Ibarra-Castanedo C., Sfarra S., Ambrosini D., Paoletti D. Bendada A., Maldague X. P., "Subsurface defect characterization in artworks by quantitative PPT," QIRT Journal, 5(2):131-149, July- Dec., 2008.

[9] Ambrosini D., Di Biase R., Paoletti D. and Quaresima R. "Design and construction of an intelligent model to investigate fresco decay," Art'05 $-8^{\text {th }}$ International Conference on Non Destructive Investigations and Microanalysis for the Diagnostics and Conservation of the Cultural and Environmental Heritage, Lecce (Italy), May 15-19, 2005.

[10] Nondestructive Handbook, Infrared and Thermal Testing, Volume 3, X. Maldague technical ed., P. O. Moore ed., 3rd edition, Columbus, Ohio, ASNT Press, 2001, 718 p.

[11] Maldague X. P. Theory and practice of infrared technology for nondestructive testing, John Wiley \& Sons, N. Y. 2001.

[12] Balageas D. L., Krapez J. C. and Cielo P., "Pulsed photothermal modeling of layered materials," Journal of Applied Physics, 59(2):348-357, 1986.

[13] Martin R. E., Gyekenyesi A. L., Shepard S. M., "Interpreting the Results of Pulsed Thermography Data," Materials Evaluation, 61(5):611-616, 2003.

[14] Pilla M., Klein M., Maldague X. and Salerno A., "New Absolute Contrast for Pulsed Thermography," Proc. QIRT 2002, D. Balageas, G. Busse, G. Carlomagno (eds.), pp. 53-58, 2002.

[15] Maldague X. P. and Marinetti S. "Pulse Phase Infrared Thermography," J. Appl. Phys., 79(5):2694-2698, 1996.

[16] Ibarra-Castanedo C. and Maldague X. "Pulsed Phase Thermography Reviewed," QIRT J., 1(1):47-70, 2004.

[17] Rajic N. "Principal component thermography for flaw contrast enhancement and flaw depth characterization in composite structures," Compos. Struct., 58:521-528, 2002.

[18] Shepard S. M., Lhota J. R., Rubadeux B. A., Ahmed T., Wang D. "Enhancement and reconstruction of thermographic NDT data", Proc. SPIE - The International Society for Optical Engineering, Thermosense XXIV, Orlando, FL, 2002, Eds. X. P. Maldague and A. Rozlosnik, 4710:531-535.

[19] Shepard S. M. "Advances in Pulsed Thermography", Proc. SPIE - The International Society for Optical Engineering, Thermosense XXVIII, Orlando, FL, 2001, Eds. A. E. Rozlosnik and R. B. Dinwiddie, 4360:511-515, 2001.

[20] Madruga F. J., Ibarra-Castanedo C., Conde O. M, Maldague X. P. and Lopez-Higuera J. M "Enhanced contrast detection of subsurface defects by pulsed infrared thermography based on the fourth order statistic moment, kurtosis," SPIE - The International Society for Optical Engineering, Thermosense XXXI, Orlando, FL, April 13-17 2009, Eds. Douglas D. Burleigh and Ralph B. Dinwiddie, 7299:72990U.

[21] Madruga F. J., Ibarra-Castanedo C., Conde O. M, Lopez-Higuera J. M and Maldague X. P. "Automatic data processing based on the skewness statistic parameter for subsurface defect detection by active infrared thermography," Proc. QIRT 9 - Quantitative Infrared Thermography, [CD-ROM], Krakow, Poland, July 2-5, 2008.

[22] Ibarra-Castanedo C., Avdelidis N. P., Grenier M., Maldague X. P. and Bendada A. "Active thermography signal processing techniques for defect detection and characterization on composite materials," Proc. SPIE - The International Society for Optical Engineering, Thermosense XXXII, Orlando, FL, April 5-9 2010, Eds. Ralph B. Dinwiddie and Morteza Safai, Paper 7661-24.

[23] Sfarra S., Ibarra-Castanedo C., Bendada A., Maldague X., Ambrosini D. and Paoletti D. "Comparative study for the nondestructive testing of advanced ceramic materials by infrared thermography and holographic interferometry," Proc. SPIE - The International Society for Optical Engineering, Thermosense XXXII, Orlando, FL, April 5-9 2010, Eds. Ralph B. Dinwiddie and Morteza Safai, Paper 7661-26.

[24] Carslaw H. S. and Jaeger J. C. Conduction of Heat in Solids, Oxford, Clarendon Press, 1959.

[25] Sfarra S., Ambrosini D., Paoletti A., Paoletti D. Ibarra-Castanedo C., Bendada A., Maldague X. P. "Quantitative Infrared Thermography (IRT) and Holographic Interferometry (HI): Nondestructive Testing (NDT) for defects detection in the Silicate Ceramics Industry," Proc. CIMTEC - International Conferences on Modern Materials and Technologies, [CD-ROM], Montecatini Terme, Tuscany, Italy, June 6-18, 2010. 